\title{
Flux density monitoring of radio sources with detected or supposed $\gamma$-ray emission
}

\author{
W. Reich ${ }^{1}$, P. Reich ${ }^{1}$, M. Pohl ${ }^{1,2}$, R. Kothes ${ }^{1}$, and R. Schlickeiser ${ }^{1}$ \\ 1 Max-Planck-Institut für Radioastronomie, Auf dem Hügel 69, D-53121 Bonn, Germany \\ 2 Max-Planck-Institut für Extraterrestrische Physik, Giessenbachstrasse, D-85740 Garching, Germany
}

Received November 26, 1997; accepted January 27, 1998

\begin{abstract}
We present the results of a flux density monitoring program of extragalactic radio sources with the Effelsberg 100-m telescope. Data for 53 sources are listed from observations between 1991 and 1995/1996 in the frequency range from $1.4 \mathrm{GHz}$ to $32 \mathrm{GHz}^{1}$. The sources have either been identified by EGRET on board CGRO at a different level of confidence or at least are candidates for showing up in the $\gamma$-ray range. The data will be useful for studies of the relationship of $\gamma$-ray activity to radio activity.
\end{abstract}

Key words: $\gamma$-rays — extragalactic radio sources quasars — BL Lacs — variability

\section{Introduction}

We have published multifrequency radio light curves of 12 extragalactic radio sources in the northern sky (Reich et al. 1993, Paper I), which have been detected by the Energetic Gamma Ray Experiment Telescope (EGRET, Kanbach 1988) on board the Compton Gamma Ray Observatory (CGRO). The data presented in Paper I cover the period from 1991 until the end of 1992 and represent peak flux densities measured in the cm-range and mm-range with the Effelsberg 100-m telescope and the IRAM 30-m telescope. From these data it was noted that enhanced radio emission is delayed up to several months to the $\gamma$-ray emission for the majority of the observed sources. Meanwhile more $\gamma$-ray detections of extragalactic sources have been reported and improved $\gamma$-ray flux densities have been derived (Fichtel et al.

Send offprint requests to: W. Reich

1 Table 2 is only available in electronic form at CDS via anonymous ftp to cdsarc.u-strasbg.fr (130.79.128.5) or via http://cdsweb.u-strasbg.fr/Abstract.html
1994; Thompson et al. 1995, 1996; Kanbach 1996; Mukherjee et al. 1997). We have continued the monitoring of extragalactic sources with the Effelsberg telescope. The relation of $\gamma$-ray activity to radio activity is of considerable interest for various reasons:

(1) First, in some $\gamma$-ray selected blazars it has been established that $\gamma$-ray flaring coincides with the launching of a new superluminal VLBI-jet component (e.g. Pohl et al. 1995; Krichbaum et al. 1995), and measuring the time delay between $\gamma$-ray flaring and radio appearance provides valuable information about the jet component formation, collimation and acceleration processes.

(2) Secondly, $\gamma$-ray loud AGN could contribute a large fraction of the diffuse extragalactic $\gamma$-ray background radiation due to the superposition of unresolved discrete sources. Most approaches (Erlykin \& Wolfendale 1995; Stecker \& Salamon 1996) relate the unknown $\gamma$-ray luminosity function of blazars to their radio luminosity function, and the justification of such proportionalities can only be tested by observing individual bright $\gamma$-ray loud and quiet radio AGN.

(3) Different theoretical models of the time and spectral evolution of flaring blazars make definite predictions about the onset of flares in different frequency bands, so that multifrequency broadband modelling provides an excellent test data set to discriminate quantitatively between different models.

A statistical analysis of the radio properties of EGRET sources and specifically the relation between radio and $\gamma$ ray emission has been published elsewhere (Mücke et al. 1996, 1997). It was found that those flat-spectrum radio sources which have been detected by EGRET also show more activity at $\mathrm{cm}$ radio wavelengths than similar sources which remain $\gamma$-ray quiet during the last years. However, there is no direct correlation between the radio and $\gamma$ ray light curves, neither in flux density nor in luminosity. Previous findings of a strict relation in luminosity can be entirely explained by the limited dynamical range of the EGRET data and selection effects inherent in the method 
of identification. The fact that about one third of the sources listed in Thompson et al. (1995) can be identified with radio-loud AGN with catalogue flux densities of $S(5 \mathrm{GHz}) \geq 1 \mathrm{Jy}$ indicates that there is a noisy luminosity relation which is further washed out by the strong variability both in $\gamma$-rays and at radio frequencies.

A time lag between $\gamma$-ray and radio outbursts was suggested for some sources on the basis of their light curves (e.g. Paper I) and the backextrapolation of the apparent motion of VLBI components. It should, however, be pointed out that there is no statistical evidence for this as a class property of all sources, which is mainly due to the limited sampling of the $\gamma$-ray light curve. Many of the promising candidates for a time lag in Paper I showed only one clear outburst in each wavelength regime (for example the BL Lac 0235+164, see Fig. 1). When adding more data from subsequent observations we get a less clear picture for some sources. An example is PKS $0528+134$ which showed a $\gamma$-ray outburst in 1991 followed by a radio outburst, another bright $\gamma$-ray outburst in 1993 again followed by a radio outburst, and then nothing peculiar at $\gamma$-rays in 1995 while subsequently the brightest ever recorded radio outburst was noted (Pohl et al. 1996). Our findings indicate that if there is a time lag between $\gamma$-ray and radio outbursts then it has to be different from outburst to outburst, at least for the well-sampled sources 0528+134, 3C 273, 3C 279, and 3C 454.3 (Mücke et al. 1998).

We have continued the observations presented in Paper I with the Effelsberg 100-m telescope of EGRET detected $\gamma$-ray sources and in addition we made observations of a few sources which have been expected to show up in the $\gamma$-ray range. For most of the sources observations have been made at irregular intervals starting in 1991 until mid of 1995. For a few sources flux density monitoring was continued until February 1996 when all observations stopped due to the track replacement of the telescope. The results of the observations are presented in tabulated form and for a few sources light curves are given in addition. For some sources radio light curves or data from Table 1 have already been published. We have included the references in Table 1.

\section{The radio observations}

Since autumn 1991 we have systematically monitored strong flat spectrum variable radio sources as potential counterparts of extragalactic CGRO sources at several frequencies with the Effelsberg 100-m telescope. The method of observations, the applied data reduction and the calibration have been already described in Paper I. In brief, the observations are made via orthogonal cross-scans and the peak flux densities have been obtained by fitting a Gaussian to the observed data.

The calibration of the data is based on 3C 286 with assumed flux densities of $14.4 \mathrm{Jy}, 10.4 \mathrm{Jy}, 7.5 \mathrm{Jy}, 4.5 \mathrm{Jy}$

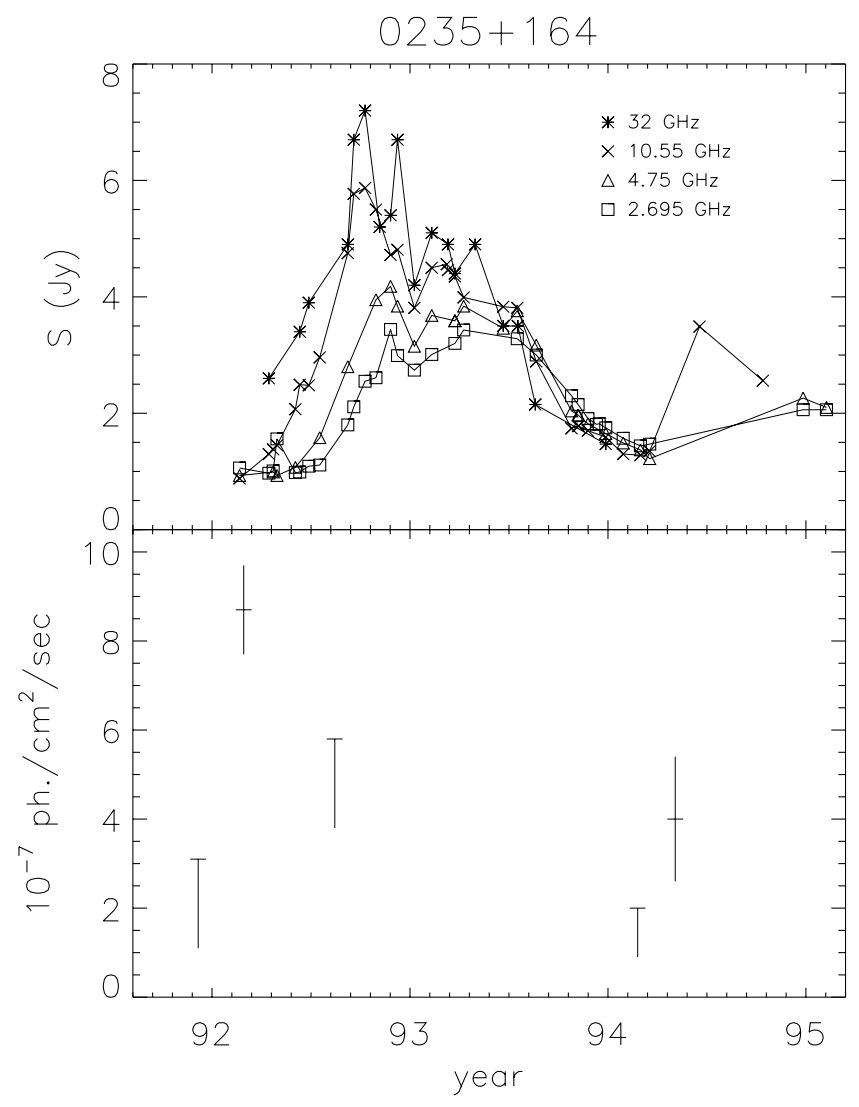

Fig. 1. Radio and $\gamma$-ray lightcurves for $0235+164$

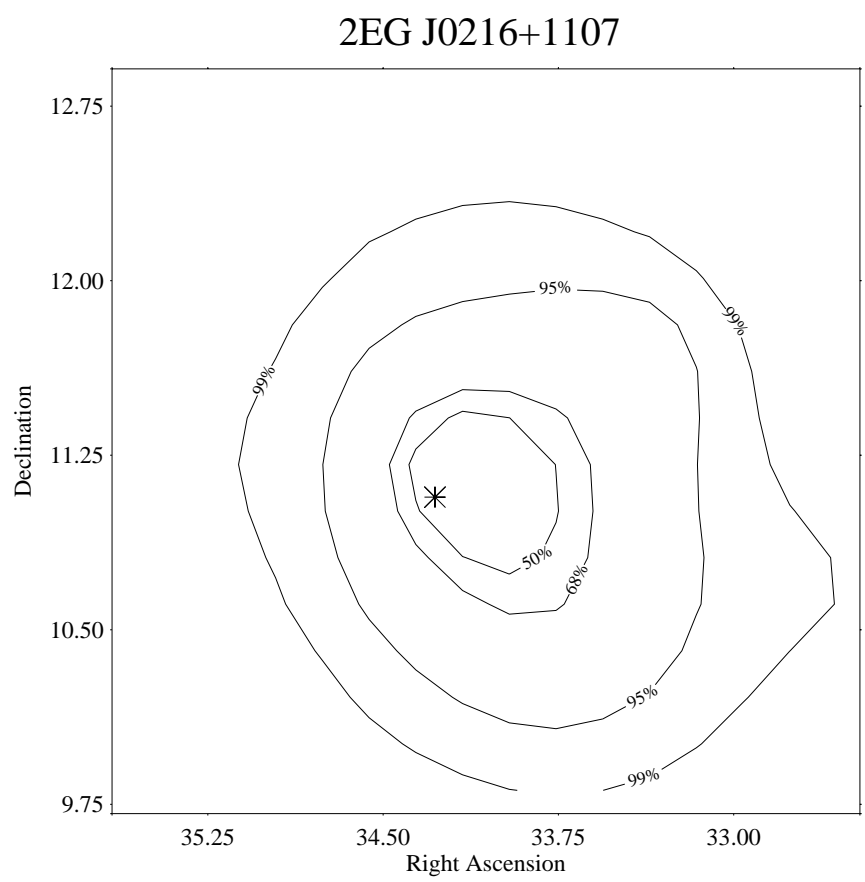

Fig. 2. Proposed identification of $0214+108$ (marked by a star) with the $\gamma$-ray source $2 \mathrm{EG}$ J0216+1107. The contours represent the statistical probability that a single source lies within the given contour 
Table 1. List of monitored radio sources

\begin{tabular}{|c|c|c|c|}
\hline Source name(s) & Epoch $19 .$. & EGRET designation/reference & Radio data/light curve \\
\hline $0130-171$ & $91.8-92.1$ & $\mathrm{E} 2 \mathrm{~m}, \mathrm{~K} 96$ & - \\
\hline $0202+149$ & $92.4-95.5$ & E1, E2, Mu97, K96 & R93 \\
\hline $0214+108$ & $92.5-95.0$ & 2EG J0216+1107 ?, Fig. 2, K96 & - \\
\hline $0219+4283 \mathrm{C} 66 \mathrm{~A}$ & $93.9-94.2$ & E2m, Mu97, K96 & - \\
\hline $0234+285$ & $91.9-95.5$ & $\mathrm{E} 1, \mathrm{E} 2 \mathrm{~m}, \mathrm{~K} 96$ & - \\
\hline $0235+164$ & $92.1-95.5$ & E1, E2, Mu97, K96 & R93, Fig. 1 \\
\hline 0336-019 СТА26 & $92.1-92.4$ & Mu97 & - \\
\hline $0420-014$ & $92.4-95.1$ & E1, E2, Mu97, K96 & R93 \\
\hline 0440-003 NRAO190 & $93.4-95.5$ & E2S, Mu97, K96 & MG97 \\
\hline $0446+112$ & $93.3-95.5$ & E1, E2, Mu97, K96 & - \\
\hline $0454-234$ & $92.4-95.1$ & E1m, K96 & - \\
\hline $0458-020$ & $92.4-94.8$ & E1m, E2m, Mu97, K96 & - \\
\hline $0528+134$ & $91.8-96.1$ & E1, E2, Mu97, K96 & R93, P95, P96 \\
\hline $0716+714$ & $92.1-95.0$ & E1, E2, Mu97, K96 & R93 \\
\hline $0735+178$ & $92.8-95.0$ & E2, Mu97 & - \\
\hline $0738+549$ & $93.6-95.1$ & 2EG J0744+5438 ?, Fig. 3, K96 & Fig. 4 \\
\hline $0804+499$ & $92.3-95.1$ & E1m, E2m, K96 & - \\
\hline $0805-077$ & $92.8-94.0$ & $\mathrm{E} 2 \mathrm{~m}$ & - \\
\hline $0827+243$ & $92.8-95.1$ & E1, E2, Mu97, K96 & - \\
\hline $0829+046$ & $92.8-93.0$ & E1m, E2, Mu97, K96 & - \\
\hline $0836+710$ & $92.0-95.0$ & E1, E2, Mu97, K96 & R93 \\
\hline $0906+4303 \mathrm{C} 216$ & $92.7-95.2$ & K96 & \\
\hline $0954+658$ & $92.0-95.0$ & E2, Mu97, K96 & - \\
\hline $1101+385$ MKN 421 & $92.3-95.2$ & E1, E2, Mu97, K96 & R93 \\
\hline $1127-145$ & $91.8-95.2$ & E2, Mu97, K96 & - \\
\hline $1156+2954 \mathrm{C} 29.45$ & $93.3-95.1$ & E2, Mu97, K96 & - \\
\hline $1219+285$ & $93.0-95.1$ & E2S, Mu97, K96 & - \\
\hline $1226+0233 \mathrm{C} 273$ & $91.2-95.2$ & E1, E2, Mu97, K96 & R93, M97 \\
\hline $1229-021$ & $93.3-95.2$ & E1m, E2, Mu97, K96 & - \\
\hline $1253-0553 \mathrm{C} 279$ & $91.8-95.6$ & $\mathrm{E} 1, \mathrm{E} 2, \mathrm{Mu} 97, \mathrm{~K} 96$ & R93, Fig. 5 \\
\hline $1406-076$ & $92.3-95.2$ & E1, E2, Mu97, K96 & - \\
\hline $1510-089$ & $91.9-95.2$ & E1, E2, Mu97, K96 & - \\
\hline $1606+106$ & $91.1-94.2$ & E1, E2, Mu97, K96 & - \\
\hline $1611+343$ & $91.1-95.1$ & E2, Mu97, K96 & Fig. 6 \\
\hline $1622-253$ & $91.9-93.2$ & E1, E2, Mu97, K96 & - \\
\hline $1622-297$ & $91.9-95.6$ & Mu97 & - \\
\hline $1633+382$ & $91.8-95.1$ & E1, E2, Mu97, K96 & - \\
\hline $1641+399$ 3C 345 & $90.9-95.2$ & - & - \\
\hline 1652+398 MKN 501 & $91.3-95.0$ & - & - \\
\hline 1730-130 NRAO530 & $91.9-95.6$ & $\mathrm{E} 2, \mathrm{Mu} 97$ & - \\
\hline $1739+522$ & $91.8-95.0$ & E2, Mu97, K96 & - \\
\hline $1741-038$ & $91.9-94.2$ & E1, K96 & - \\
\hline $1823+018$ & $91.8-92.1$ & K96 & - \\
\hline $2200+420$ BL LAC & $92.3-93.6$ & Mu97 & - \\
\hline 2230+114 CTA102 & $92.3-95.6$ & E1, E2, Mu97, K96 & R93 \\
\hline $2251+1583 \mathrm{C} 454.3$ & $92.1-95.8$ & E1, E2, Mu97, K96 & R93, A98 \\
\hline $2356+196$ & $92.5-95.5$ & E1m, E2, Mu97, K96 & - \\
\hline
\end{tabular}

$\mathrm{E} 1=$ first EGRET catalogue, Fichtel et al. (1994), E1m = marginal detections

$\mathrm{E} 2=$ second EGRET catalogue, Thompson et al. (1995), E2m = marginal identification

E2S = supplement to the second EGRET catalogue, Thompson et al. (1996)

Mu97 = Mukherjee et al. (1997)

A98 = Aller et al. (1998)

K96 = Kanbach (1996)

M97 = von Montigny et al. (1997)

MG97 = McGlynn et al. (1997)

P95 = Pohl et al. (1995)

P96 = Pohl et al. (1996)

R93 = Reich et al. (1993, Paper I). 
Table 3. Single measurements

\begin{tabular}{llllll}
\hline Source name(s) & Epoch 19.. & EGRET reference & $S(2.695 \mathrm{GHz})$ & $S(4.75 \mathrm{GHz})$ & $S(10.45 \mathrm{GHz})$ \\
\hline $0115+027$ 3C 37 & 93.025 & K96 & $0.89 \pm 0.04$ & $0.61 \pm 0.02$ & $0.51 \pm 0.04$ \\
$0430+290$ & 93.271 & K96 & $0.47 \pm 0.03$ & $0.50 \pm 0.03$ & $0.44 \pm 0.03$ \\
$0917+449$ & 92.500 & E2, Mu97, K96 & $1.21 \pm 0.08$ & $1.32 \pm 0.07$ & $1.74 \pm 0.12$ \\
$0954+556$ & 93.331 & E2, Mu97, K96 & $2.43 \pm 0.27$ & $1.95 \pm 0.13$ & $1.45 \pm 0.13$ \\
$1222+216$ & 93.027 & E2, Mu97, K96 & $1.36 \pm 0.05$ & $1.13 \pm 0.04$ & $1.73 \pm 0.09$ \\
$2022-077$ & 93.944 & E2, K96 & $0.95 \pm 0.03$ & - & $0.70 \pm 0.10$ \\
\hline
\end{tabular}

The EGRET references are as for Table 1.

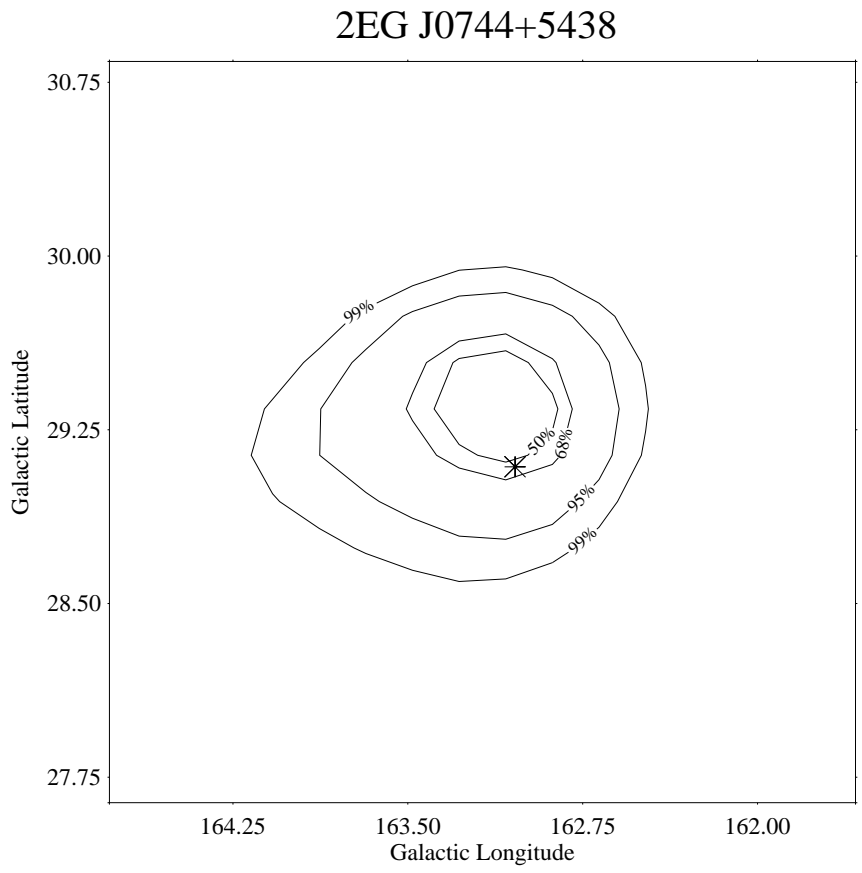

Fig. 3. Proposed identification of $0738+545$ (marked by a star) with the $\gamma$-ray source 2 EG J0744+5438. The contours represent the statistical probability that a single source lies within the given contour

and 2.15 Jy at $1.4 \mathrm{GHz}, 2.695 \mathrm{GHz}, 4.75 \mathrm{GHz}, 10.55 \mathrm{GHz}$ and $32 \mathrm{GHz}$, respectively. 3C 138 served as a secondary calibrator and its flux density was measured every few months in respect to $3 \mathrm{C} 286$ because of its weak variability. Occasionally also NGC 7027, 3C 48 and 3C 295 have been used as secondary calibrators in respect to $3 \mathrm{C} 286$. The data obtained at other frequencies than those listed have been calibrated by interpolation of the flux densities of 3C 286. The Effelsberg 100-m telescope has an angular resolution (HPBW) of 9.4 at $1.4 \mathrm{GHz}, 4.3$ at $2.695 \mathrm{GHz}$, 2 '.4 at $4.75 \mathrm{GHz}, 1.15$ at $10.55 \mathrm{GHz}$ and 0.45 at $32 \mathrm{GHz}$. The $1.4 \mathrm{GHz}$ receiver (tunable up to $1.7 \mathrm{GHz}$ ) is installed in the primary focus of the telescope, while the $2.695 \mathrm{GHz}$, $4.75 \mathrm{GHz}, 10.55 \mathrm{GHz}$ and $32 \mathrm{GHz}$ receivers are located in the secondary focus.

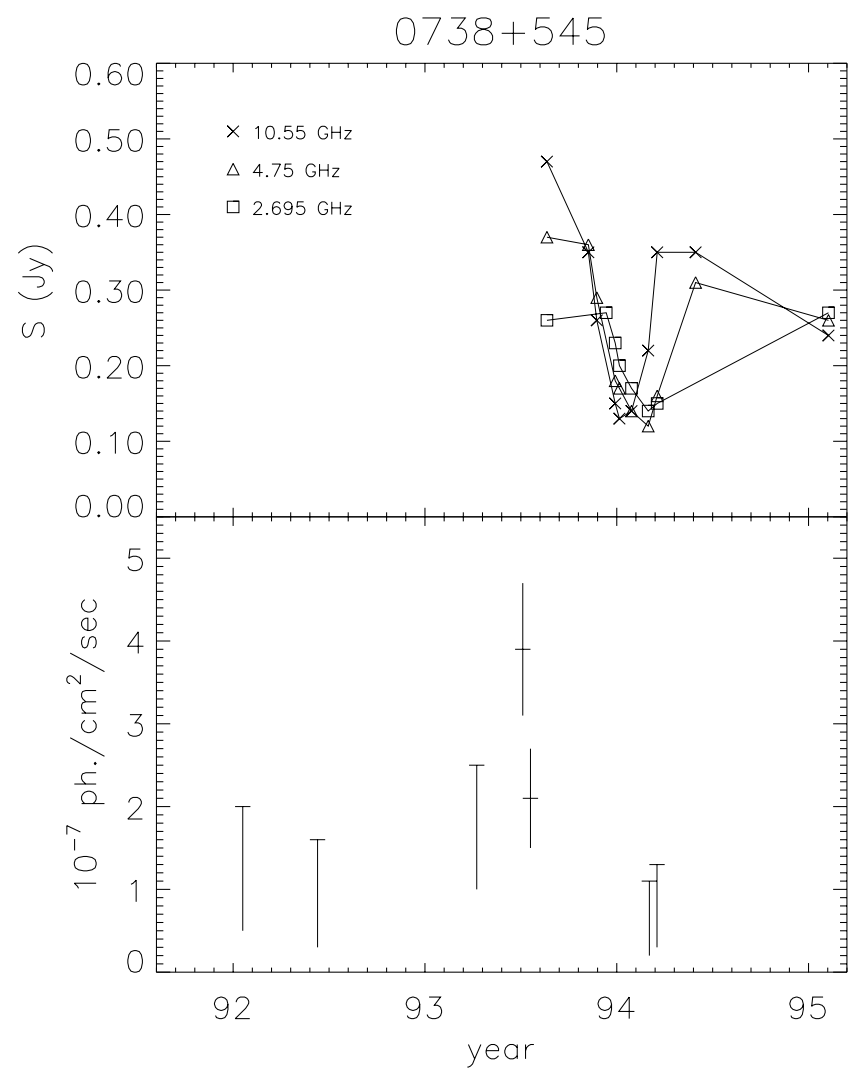

Fig. 4. Radio and $\gamma$-ray lightcurves for $0738+545$. The radio source shows remarkable spectral changes on short time scales

The occasionally used receivers at $2.3 \mathrm{GHz}$ and 8.6 $\mathrm{GHz}$ are also installed in the secondary focus, while the maser receiver operating in the $22 / 23 \mathrm{GHz}$ band, the $3.3 \mathrm{GHz}$ and $30 \mathrm{GHz}$ receivers are mounted in the prime focus. More details about the different receiving systems have been compiled by Schmidt \& Zinz (1994).

The $4.75 \mathrm{GHz}$ receiver has been replaced by a new 4.85 GHz receiving system in August 1995. A shift of the centre frequency from $10.55 \mathrm{GHz}$ to $10.45 \mathrm{GHz}$ was made in early 1995 due to severe interference caused by the TVsatellite ASTRA $1 \mathrm{D}$. 


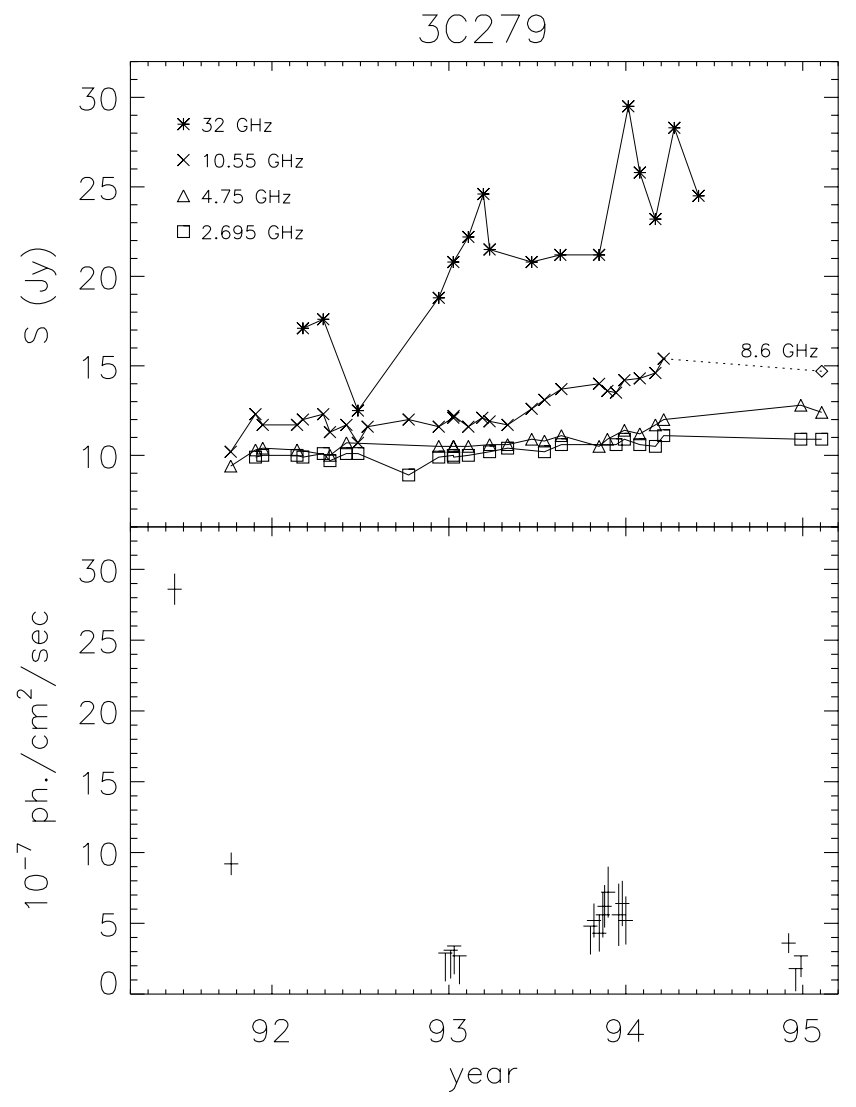

Fig. 5. Radio and $\gamma$-ray lightcurves for $3 \mathrm{C} 279$

\section{Results}

\subsection{Tabulated data}

In Table 1 we have listed the sources monitored and the epoch of observations. Table 2 contains the peak flux densities and the calculated errors. Table 2 is available only in electronic form at the CDS, Strasbourg via anonymous ftp 130.79 .128 .5 or via http://cdsweb.ustrasbg.fr/Abstract.html.

In Table 2 we list the day of the observation, the frequency, peak flux density and error. The peak flux has been calculated from the Gaussian fit of the two orthogonal scans observed and is corrected for residual pointing errors of the telescope. The pointing corrected amplitudes are derived for each scanning direction independently and are averaged. The error includes uncertainties from the Gaussian fit parameters and the pointing results. The maximum error from both results is taken for the averaged amplitude. We convert the data into flux densities by using the mean of scaling factors found from all the observed calibrators during an observing session. We used the maximum and minimum of all these scaling factors and add the resulting flux density changes to the error. By this approach the resulting error includes a number of time dependent effects like changes in opacity, focal changes or gain variations of the antenna during an observing run. To

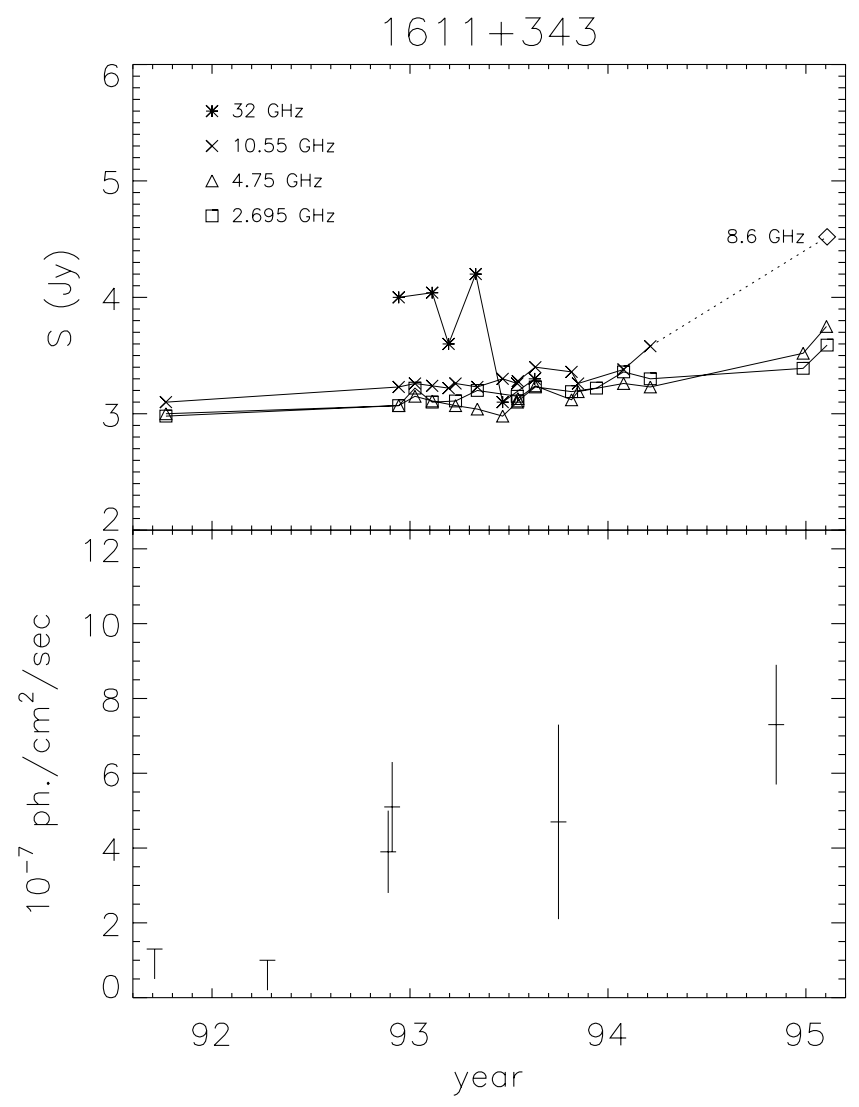

Fig. 6. Radio and $\gamma$-ray lightcurves for $1611+343$

take these effects into account is of particular importance when variability is under investigation. We note, however, that the errors resulting by our approach are systematically higher than those quoted from other flux density monitoring programs.

\subsection{Comments on individual sources}

Our sample includes two radio sources which have not been taken as identification for the respective $\gamma$-ray sources in the EGRET catalogues, but included in the list of Kanbach (1996). The association of $0738+549$ with 2 EG J0744+5438 has already been pointed out by Mukherjee et al. (1995). We also regard $0214+108$ as a likely identification for 2EG J0216+1107. In Figs. 2 and 3 we show likelihood finemaps of both $\gamma$-ray sources (taken from Thompson et al. 1995) with the position of the radio source indicated. Both radio sources have also been listed as potential EGRET sources by Mattox et al. (1997) who used Bayesian statistics to evaluate the probability for a correct cross-identification of $\gamma$-ray and radio sources.

A few examples of radio and $\gamma$-ray light curves are shown for $0235+164$ (Fig. 1), 0738+545 (Fig. 4), 3C 279 (Fig. 5) and 1611+343 (Fig. 6) in order to illustrate the rather different variability behaviour of sources in the sample. In general, the sampling of the $\gamma$-ray light curve 
is rather coarse if compared to the radio light curves and much short-term variability remains undetected. This needs to be taken into account when comparing the variability behaviour in both wavelength ranges.

We have added observations of MKN 501 in Table 1 which has not been identified as an EGRET source but has recently been seen at $\mathrm{TeV} \gamma$-ray energies with the Whipple Cherenkov telescope (Quinn et al. 1996) or the HEGRA Cherenkov telescope (Bradbury et al. 1997). Also data for 3C 345 are included, since its strength in flux density and its high variability make it a potential $\gamma$-ray source candidate.

\subsection{Single radio measurements}

In Table 3 we list flux densities for six sources which have been observed once. Most of the data are from a program to observe quasi simultaneously the spectra of radio sources with soft X-rays counterparts as revealed by ROSAT (Reich et al., in preparation). We note that most of the sources listed in Table 1 are visible in the ROSAT all-sky survey.

\section{Conclusion}

We have observed flux densities of 53 extragalactic radio sources with the Effelsberg 100-m telescope in the frequency range from $1.4 \mathrm{GHz}$ to $32 \mathrm{GHz}$ around the time of detection by EGRET on board CGRO and afterwards until the end of 1995 or the beginning of 1996 . Nearly 2500 individual measurements have been made. The results are available in electronic form.

The radio database will be helpful to clarify the relation of the $\gamma$-ray variability to the radio variability. First studies by Mücke et al. $(1996,1997)$ indicate that the correlation of the light curves is rather complex and a statistical analysis needs to take into account the coarse sampling and limited dynamic range of the EGRET data.

Acknowledgements. We thank the Effelsberg telescope operators for qualified support.

\section{References}

Aller M.F., Marscher A.P., Hartman R.C., et al., 1998, in: Dermer C.D., Kurfess J. (eds.) Proc. 4th Compton Symposium, AIP Conf. Ser. (in press)

Bradbury S.M., Deckers T., Petry D., et al., 1997, A\&A 320, L5

Erlykin A.D., Wolfendale A.W., 1995, J. Phys. G. 21, 1149

Fichtel C.E., Bertsch D.L., Chiang J., et al., 1994, ApJS 94, 551

Kanbach G., 1988, Sp. Sci. Rev. 49, 69

Kanbach G., 1996, in: Kirk J.G., Camenzind M., von Montigny C., Wagner S. (eds.) Proc. of the Heidelberg Workshop on the Gamma-Ray Emitting AGN. Max-Planck-Institut für Kernphysik, Heidelberg, p. 1

Krichbaum T.P., Britzen S., Standke K.J., et al., 1995, in: Cohen M., Kellermann K. (eds.) Quasars and AGN. Proc. Nat. Acad. Sci. 92, p. 11377

Mattox J.R., Schachter J., Molnar L., et al., 1997, ApJ 481, 95

McGlynn Th.A., Hartman R.C., Bloom S.D., et al., 1997, ApJ 481,625

Mücke A., Pohl M., Reich P., et al., 1996, A\&AS 120, C541

Mücke A., Pohl M., Reich P., et al., 1997, A\&A 320, 33

Mücke A., Pohl M., Kanbach G., et al., 1998, Ap\&SS, Proc. of the Workshop "Blazars, black holes and jets", Kidger M. et al. (eds.) (in press)

Mukherjee R., Aller H.D, Aller M.F., et al., 1995, ApJ 445, 189

Mukherjee R., Bertsch D.L., Bloom S.D., et al., 1997, ApJ 490, 116

Pohl M., Reich W., Krichbaum T.P., et al., 1995, A\&A 303, 383

Pohl M., Reich W., Schlickeiser R., et al., 1996, A\&AS 120, C529

Quinn J., Akerlof C.W., Biller S., et al., 1996, ApJ 456, L83

Reich W., Steppe H., Schlickeiser R., et al., 1993, A\&A 273, 65

Schmidt A., Zinz W., 1994, Technischer Bericht Nr. 67-5, MPIfR, Bonn (update: http://www.mpifrbonn.mpg.de/w3/technik/rxmain.html)

Stecker F.W., Salamon M.H., 1996, ApJ 464, 600

Thompson D.J., Bertsch D.L., Dingus B.L., et al., 1995, ApJS 101,259

Thompson D.J., Bertsch D.L., Dingus B.L., et al., 1996, ApJS 107,227

von Montigny C., Aller H., Aller M., et al., 1997, ApJ 483, 161 